\title{
Condensation phase transitions of symmetric conserved-mass aggregation model on complex networks
}

\author{
Sungchul Kwon, Sungmin Lee, and Yup Kin* \\ Department of Physics and Research Institute for Basic Sciences, Kyung Hee University, Seoul 130-701, Korea
}

(Dated: November 12, 2018)

\begin{abstract}
We investigate condensation phase transitions of symmetric conserved-mass aggregation (SCA) model on random networks (RNs) and scale-free networks (SFNs) with degree distribution $P(k) \sim$ $k^{-\gamma}$. In SCA model, masses diffuse with unite rate, and unit mass chips off from mass with rate $\omega$. The dynamics conserves total mass density $\rho$. In the steady state, on RNs and SFNs with $\gamma>3$ for $\omega \neq \infty$, we numerically show that SCA model undergoes the same type condensation transitions as those on regular lattices. However the critical line $\rho_{c}(\omega)$ depends on network structures. On SFNs with $\gamma \leq 3$, the fluid phase of exponential mass distribution completely disappears and no phase transitions occurs. Instead, the condensation with exponentially decaying background mass distribution always takes place for any non-zero density. For the existence of the condensed phase for $\gamma \leq 3$ at the zero density limit, we investigate one lamb-lion problem on RNs and SFNs. We numerically show that a lamb survives indefinitely with finite survival probability on RNs and SFNs with $\gamma>3$, and dies out exponentially on SFNs with $\gamma \leq 3$. The finite life time of a lamb on SFNs with $\gamma \leq 3$ ensures the existence of the condensation at the zero density limit on SFNs with $\gamma \leq 3$ at which direct numerical simulations are practically impossible. At $\omega=\infty$, we numerically confirm that complete condensation takes place for any $\rho>0$ on RNs. Together with the recent study on SFNs, the complete condensation always occurs on both RNs and SFNs in zero range process with constant hopping rate.
\end{abstract}

PACS numbers: 05.70.Fh,05.40.-a,89.75.Da,89.75.Hc

\section{INTRODUCTION}

Nonequilibrium condensation phase transitions from fluid phase into condensed phase have been observed in a variety of phenomena ranging from traffic flow to polymer gels 1, 2, 3, 4, 5, 6, 7, 8, 9, 10, 11, 12, 13. In the steady state, a finite fraction of total particles condenses on a single site in condensed phase when the total particle density $\rho$ is increased beyond a certain critical value $\rho_{c}$. In fluid phase below $\rho_{c}$, the particle number of each site fluctuates around $\rho$ without the condensation.

Various kinds of nonequilibrium mass transport models exhibit the condensation transitions or only condensation. The simplest and well-known model is zero-range process(ZRP) in one-dimension [1]. In ZRP, many identical particles occupy sites on a lattice. Each site may contain an integer number of particles and one of these particles can hop to one of the nearest neighboring sites with a rate that depends on the number of particles at the site of departure. The chipping (single-particle dissociation) and aggregation processes of ZRP describe various condensations such as jamming of traffic [2], bunching of buses [3], coalescence of shaken steel balls [4] and condensation of edges in networks [5]. Recent studies of ZRP on scale-free [ $\underline{6}]$ and directed networks [7] reveal the conditions under which condensation takes place.

Another important class of condensation transitions emerges when the diffusion of the whole particles of a

*Electronic address: ykim@khu.ac.kr single site is involved in addition to the chipping and aggregation. These processes arise in a variety of phenomena such as polymer gels 8], the formation of colloidal suspensions [9], river networks [10, 11] and clouds [12]. Recently studied conserved-mass aggregation $(\mathrm{CA})$ model is the simplest one incorporating diffusion, chipping and aggregation upon contact [13, 14]. In one dimensional CA model, the mass $m_{i}$ of site $i$ moves either to site $i-1$ or to site $i+1$ with unit rate, and then $m_{i} \rightarrow 0$ and $m_{i \pm 1} \rightarrow m_{i \pm 1}+m_{i}$. With rate $\omega$, unit mass chips off from site $i$ and moves to one of the nearest neighboring sites; $m_{i} \rightarrow m_{i}-1$ and $m_{i \pm 1} \rightarrow m_{i \pm 1}+1$. As total masses are conserved, the conserved density $\rho$ and $\omega$ determine the phase of CA model. The condensation transition arises via the competition between diffusion and chipping processes. The diffusion of masses tends to produce massive aggregates and consequently creates more vacant sites. The chipping of unit mass tends to prevent the formation of aggregates, so that it leads to a replenishment of the lower end of mass distribution.

The single site mass distribution $P(m)$, i.e., the probability that a site has mass $m$ in the steady state, was shown to undergo phase transitions on regular lattices [13. For a fixed $\omega$, as $\rho$ is varied across the critical value $\rho_{c}(\omega)$, the behavior of $P(m)$ for large $m$ was found to be 13 .

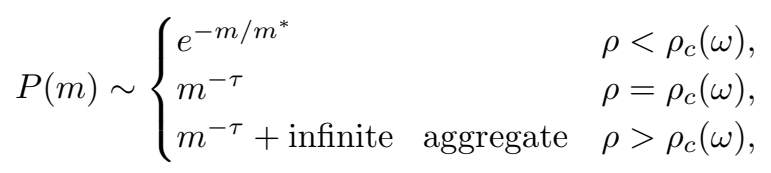

where $\rho_{c}$ is given as $\rho_{c}(\omega)=\sqrt{\omega+1}-1 . \rho_{c}$ and $\tau$ are shown to be independent of spatial dimension $d$ [14]. The 
tail of the mass distribution changes from exponential to an algebraic decay as $\rho$ approaches $\rho_{c}$ from below. As one further increases $\rho$ beyond $\rho_{c}$, this asymptotic algebraic part of the critical distribution remains unchanged but in addition an infinite aggregate forms. This means that all the additional mass $\left(\rho-\rho_{c}\right) L^{d}$ condenses onto a single site and does not disturb the background critical distribution. The $\omega=\infty$ case corresponds to ZRP with constant chipping rate, and then there is no condensation transitions on regular lattices. The critical exponent $\tau$ is same everywhere on the critical line $\rho_{c}(\omega)$. Recent studies showed that $\tau$ depends on the symmetry of movement and constraints of diffusion rate [15, 16]. In what following, we only consider the symmetric CA (SCA) model where diffusion and chipping directions are unbiased [13].

As mean field theory correctly predicts the phase diagram and $\tau$ of SCA model in one dimension [13], the nature of the condensation transitions should remain unchanged in any higher dimensions. However recent studies of various dynamics on network structures showed that the structure of networks leads to more rich and intriguing behavior different from that predicted by the standard mean-field theory on regular lattices [5, 6, 17]. For example, ZRP on SFNs was shown to exhibit condensation even at constant hopping rate which is the case of an infinite chipping rate of SCA model [6].

In this paper, we investigate the effect of network structures on the condensation transitions of SCA model using random networks (RNs) and scale-free networks (SFNs). As we shall see, on RNs and SFNs with the degree exponent $\gamma>3$, SCA model undergoes the same type of condensation transitions as those in regular lattice across a critical line $\rho_{c}(\omega)$ in $\rho$ - $\omega$ plane with the exponent $\tau=5 / 2$. However on SFNs with $\gamma \leq 3$ where one or several nodes, so-called hub nodes, have a finite fraction of links, the fluid phase completely disappears and the condensation with exponentially decaying background mass distribution takes place for any non-zero density. The out-line of this paper is as follows. In Sec. II, we introduce SCA model on complex networks. The condensation transitions on RNs and SFNs are discussed in Sec. III and IV. To understand the condensation on SFNs with $\gamma \leq 3$, we discuss lamb-lion problems on SFNs in Sec. V. In Sec. VI and VII, we discuss SCA model at $\omega=\infty$ and the effect of diffusion of masses on average mass distribution on degrees respectively. Finally we summarize our results in Sec. VIII.

\section{SCA MODEL ON NETWORKS}

We consider a network with $N$ nodes and $K$ links. The degree $k_{i}$ of a node $i$ is defined as the number of its links connected to other nodes. The average degree of a node $\langle k\rangle$ is given as $\langle k\rangle=2 K / N$. The degree distribution $P(k)$ is a Poisson distribution for RNs and a power-law distribution of $P(k) \sim k^{-\gamma}$ for SFNs. Each node may have an integer number of particles, and the mass of a node is defined as the number of particles at the node. Initially $M$ particles randomly distribute on $N$ nodes with given conserved density $\rho=M / N$. Next a node $i$ is chosen at random and one of the following events is occurred:

(i) Diffusion: With unit rate, the mass $m_{i}$ moves to the randomly selected nearest neighboring node $j$. If the node $j$ has already mass $m_{j}$, then the aggregation takes place; $m_{i} \rightarrow 0$ and $m_{j} \rightarrow m_{j}+m_{i}$.

(ii) Chipping: With rate $\omega$, unit mass (a single particle) at node $i$ chips off and moves to the randomly selected nearest neighboring node $j ; m_{i} \rightarrow m_{i}-1$ and $m_{j} \rightarrow m_{j}+1$.

As mentioned in Sec. I, diffusion and chipping processes compete each other so a condensation transition physically depends on two external parameters $\rho$ and $\omega$. At $\omega=0$, however, only diffusion and aggregation take place so masses always condense onto a single site (complete condensation). On the other hand, the $\omega=\infty$ case corresponds to ZRP with constant chipping rate. It was shown that ZRP on scale-free networks exhibits complete condensation when mass-dependent chipping rate $u(m)$ is given as $u(m) \sim m^{\delta}$, but $\delta<\delta_{c}$, where $\delta_{c}$ is some threshold value [6]. Hence the SCA model of $\omega=\infty$ is just the $\delta=0$ case of ZRP so the complete condensation always aries at $\omega=\infty$. As we shall see in the following section, the complete condensation also takes place on random networks at $\rho>0$.

For the construction of SFN, we use a static model 18 instead of preferential attachment algorithm [5]. In static model, it is desired to use large $\langle k\rangle$ to construct fully connected networks. In simulations, we use $<k>=4$.

\section{SCA MODEL ON RNS AND SFNS WITH} $\gamma>3$

We consider SCA model on RNs and SFNs with $\gamma>3$ having nodes $N=10^{4}$ and links $K=2 \times 10^{4}$ for $\omega \neq \infty$. We define the single node mass distribution $P(m, t)$ at time $t$, i.e., the probability that a node has mass $m$ at $t$ as

$$
P(m, t)=\frac{1}{N} n(m, t)=\frac{1}{N} \sum_{i=1}^{N} \delta_{m_{i}(t), m},
$$

where $n(m, t)$ is the number of nodes with mass $m$ at time $t$ and $m_{i}(t)$ is the mass of node $i$ at $t$. From the normalization condition of $P(m, t)$, i.e., $\sum_{m} P(m, t)=1$, all $P(m, t)$ should reach the stationary state at the same time. For instance, $P(m=0, t)$ is given as $P(0, t)=$ $1-\sum_{m \neq 0} P(m, t)$. Hence, if $P(0 . t)$ saturates to its steady state value $P(0)$ after some time $\tau_{0}$, then $P(m, t)$ for any mass $m$ also should reach its steady state value $P(m)$ after the time $\tau_{0}$. So the characteristic time $\tau_{m}$ at which $P(m, t)$ saturates is expected to be independent of mass $m$, and

$$
\tau_{0}=\tau_{1}=\cdots=\tau_{m^{*}}
$$


where $m^{*}$ is the maximal mass. In Fig. 1 , we plot $P(m, t)$ of $m=1$ and 10 for $\omega=1.0$ and $\rho=0.1$ on various networks. The vertical dotted line denotes the characteristic time $\tau_{m}$, which supports Eq. (3).

We measure the steady state distribution $P(m)$ by averaging $P(m, t)$ after $\tau_{m}$. Fig. 2 shows the plots of $P(m)$ versus $m$ for RN and SFN with $\gamma=4.3$ with $\omega=1.0$. The critical density $\rho_{c}$ is $\rho_{c}(\omega=1)=0.31(3)$ for $\mathrm{RN}$ and $0.24(3)$ for SFN with $\gamma=4.3$ respectively. In fluid phase $\left(\rho<\rho_{c}\right), P(m)$ exponentially decays for large mass in both RN and SFN with $\gamma=4.3$. At $\rho=\rho_{c}, P(m)$ algebraically decays on both RN and SFN with $\gamma=4.3$ as $P(m) \sim m^{-\tau}$ with $\tau=2.38(3)$ for RN and $\tau=2.33(2)$ for SFN with $\gamma=4.3$ without condensations. The values of $\tau$ of both networks also agree well with $\tau=5 / 2$ of regular lattices [13, 14]. In condensed phase $\left(\rho=3.0>\rho_{c}\right)$, the access mass of $\left(\rho-\rho_{c}\right) N \approx 2.7 \times 10^{4}$ condenses on a single node in the both networks without changing the background critical distribution. To map out phase diagrams for RN and SFN with $\gamma=4.3$, we measure $\rho_{c}$ and $\tau$ for $\omega=0.1,1.0$ and 10 , and find that $\tau$ is the same within error on the critical line. Fig. 3 shows the phase diagrams of RN and SFN with $\gamma=4.3$. The solid line denotes the critical line, $\rho_{c}=\sqrt{\omega+1}-1$ of regular lattices [13]. On regular lattices, the $\rho_{c}(\omega)$ is independent of dimensionality. However on networks, $\rho_{c}(\omega)$ depends on the underlying network structures. We also confirm that SFN with $\gamma=3.5$ undergoes the same type of condensation transitions. Therefore we conclude that SCA model on RNs and SFNs with $\gamma>3$ undergoes the same type of condensation transitions from fluid phase into condensed phase for $0<\omega<\infty$ as those on one-dimensional regular lattice. However the critical line depends on the underlying network structures.

\section{SCA MODEL ON SFN WITH $2 \leq \gamma \leq 3$}

For $\omega \neq \infty$, we perform simulations on SFNs with $\gamma=2.4$ and 3 with $N=10^{4}$ nodes and $K=2 \times 10^{4}$ links. We plot $P(m, t)$ of $\gamma=2.4$ in Fig. 1 (c) for example, and average $P(m, t)$ after $\tau_{m}$ for the steady state $P(m)$. As mentioned in the previous section, $\tau_{m}$ is same for all masses.

The mass distribution $P(m)$ for $\gamma \leq 3$ show quite different behavior from that for $\gamma>3$. Fig. 4 shows $P(m)$ on SFNs with $\gamma=2.4$ and 3 for $\rho=0.2$ and 3.0 with $\omega=1$. As in the condensed phase of RNs and SFNs with $\gamma>3$, there is a condensation of mass $m^{*} \approx \rho N$. However $P(m)$ exponentially decays for large mass rather than power-law. We also measure $P(m)$ for various $\omega$ and $\rho$, and confirm the same behavior of $P(m)$. Therefore we conclude that for $\omega \neq \infty$, SCA on SFNs with $\gamma \leq 3$ exhibits an infinite aggregation with an exponential background mass distribution. We call this phase incomplete condensed phase to reflect exponential mass distributions (Fig. 4 (c)). Intriguingly the two features of the fluid and the condensed phase on regular lattice coexist on SFNs with $\gamma \leq 3$. Such a behavior was found in the lattice gas model proposed to describe the distribution of droplets in the fragmentation process following a nuclear collision 22].

The nonexistence of the fluid phase for $\gamma \leq 3$ mainly comes from the hub structure of SFNs with $\gamma \leq 3$ where one or several nodes have a finite fraction of total links 5]. Due to the hub structure, all masses move to a hub node by both diffusion and chipping processes unlike SFNs with $\gamma>3$ where the chipping tends to split masses and prevents aggregations. Hence there are no processes to prevent the formation of infinite aggregation for $\gamma \leq 3$. The difference from ZRP is that the diffusion process moves an infinite aggregation of mass $m_{h u b}$ at a hub node to the others. Then the chipping distributes small masses of $m_{h u b}$ onto neighboring nodes. In this way, nodes with small degree can have mass so the resultant $P(m)$ is exponential.

It is hard to show the existence of infinite aggregation for the limit of $\rho \rightarrow 0$ numerically with finite size networks. Instead we investigate annihilating random walks of two particles on SFNs (lamb-lion problem) for the condition of the existence of the fluid phase on SFNs. For $\rho \rightarrow 0$ limit, we assume an infinite aggregation and neglect the presence of other masses. Then unit mass chips off from the aggregation, and diffuses around on the networks. For the existence of an infinite condensate in the steady state, the two masses should aggregate again in finite time interval. If not, unit mass continuously chips off from the infinite aggregation, which will finally disappear. Hence if the unit mass (lamb) survives indefinitely without meeting the infinite aggregation (lion), then the fluid phase exists at zero density limit. Otherwise, the condensed phase exists for any $\rho>0$. In next section, we investigate a lamb-lion problem on RNs and SFNs.

\section{LAMB-LION PROBLEM ON NETWORKS}

We consider one lamb and one lion problem on networks. If a lamb meets a lion on the same node, then it dies. Hence the interesting quantity is the survival probability $S(t)$ of a lamb at time $t$. On $d$ dimensional regular lattice, $S(t)$ depends on $d$ as follows $23,24,25,26]$.

$$
S(t) \sim\left\{\begin{array}{l}
t^{d / 2-1} \quad \text { for } d<2 \\
1 / \ln t \quad \text { for } d=2 \\
\text { constant for } d>2
\end{array}\right.
$$

On networks, the steady state distribution of a random walker is proportional to the degree $k_{i}$ of node $i[19]$ as

$$
P_{i}^{\infty}=k_{i} / \sum_{j=1}^{N} k_{j} .
$$

Hence the probability of finding two walkers at the same node should depend on the second moment $<k^{2}>$. While $<k^{2}>$ is finite for RNs and SFNs with $\gamma>3$, 
it diverges for SFNs with $\gamma \leq 3$. So RNs and SFNs with $\gamma>3$ correspond to infinite dimensional homogeneous regular lattice in macroscopic scale, while SFNs with $\gamma \leq 3$ are generically inhomogeneous. Therefore $S(t)$ is expected to be constant in the thermodynamic limit on RNs and SFNs with $\gamma>3$. It means the existence of the fluid phase for the limit of $\rho=0$ for any $\omega \neq \infty$ as shown in Fig. 3. On the other hand, for the incomplete condensed phase of $\gamma \leq 3, S(t)$ should decay to zero for $\gamma \leq 3$.

We measure $S(t)$ of a lamb on RNs and SFNs with various $\gamma$ for several values of $N$ from $10^{3}$ to $10^{6}$ and up to $10^{6}$ time steps. Initially a lamb and a lion are placed on randomly selected two nodes. In Fig. 5 , we plot $S(t)$ for $\gamma=4.3$ (a) and 2.4 (b) for various system size $N$. Interestingly $S(t)$ decays exponentially in both SFNs for any $N$. For example, $S(t)$ of $\gamma=4.3$ and $N=10^{6}$ exponentially decays with very large characteristic time $\tau$ order of $10^{6}$ over the whole time interval. We also confirm the same exponential decay of $S(t)$ on RNs for any $N$. Unlike on regular lattices where the exponential decay of $S(t)$ comes from finite size effect of lattices, $S(t)$ on networks shows such a exponential decay at very early time. The exponential decay of $S(t)$ mainly comes from the small world nature of networks [5], i.e., the average path length between a pair of nodes increases logarithmically with $N$. As $S(t)$ shows the exponential decay for any $\gamma$, we are interested in the average life time $T$ of a lamb rather than $S(t)$ itself.

We measure the life time $T$ of a lamb on RNs and SFNs with several $\gamma$ values for $N$ up to $10^{6}$ and plot $T$ against $N$ in Fig. 5 (c) and (d). In Fig. 5 (c), $T$ linearly increases with $N$ for both RN and SFN with $\gamma=4.3$. Assuming $T \sim N^{\alpha}$, we estimate $\alpha=1.0(2)$ for RN and SFN with $\gamma=4.3$ by measuring slopes. However for $\gamma<3$ of Fig. 5 (d), $T$ exhibits quit different behavior. $T$ of $\gamma=2.15$ and 2.4 tend to saturate to some asymptotic value with decreasing successive slopes. However the characteristic size $N_{s}(\gamma)$ at which $T$ begins to saturate increases as $\gamma$ approaches $\gamma=3$. For example, $T$ of $\gamma=2.75$ seems to algebraically increase up to $N=10^{6}$. It means that $N_{s}$ of $\gamma=2.75$ is already larger than $10^{6}$. To see the saturation at $\gamma=2.75$, big size $N$ much larger than $10^{6}$ is needed but it is practically very difficult. It is hard to believe that $T$ diverges in power-law fashion as $\gamma$ approaches 3 because $T$ of small $\gamma$ is already begin to saturate at moderate $N$ order of $10^{5} \sim 10^{6}$. Hence $T$ of $\gamma<3$ is expected to saturate to its asymptotic value even though we cannot see the saturation via simulations as $\gamma$ approaches 3 from below. At $\gamma=3, T$ also increases algebraically with $N$. By measuring successive slopes, we estimate $\alpha=0.85(1)$ for $\gamma=3$. However, as the exponent $\alpha$ continuously varies as $\gamma$ increases to 3, the value of $\alpha$ at $\gamma=3$ may have no special meaning. As shown in Fig. 4 (a) for $\gamma=3$, the condensation already occurs at sufficiently low density $\rho=0.2$ even for small size $N=10^{4}$ so $T$ of $\gamma=3$ is believed to saturate in thermodynamic limit as for $\gamma=2.75$. Therefore we are convinced that the life time of a lamb is finite in thermodynamic limit of $N \rightarrow \infty$ for $\gamma \leq 3$. From the behavior of the life time $T$, the asymptotic behavior of $S(t)$ is investigated in the following.

As shown in Fig. 5 (a), $S(t)$ decays exponentially as $S(t)=S_{o} e^{-t / \tau}$. From the definition of $T=$ $\int_{0}^{\infty} t(-d S(t) / d t) d t$, we have $T \sim \tau$. Hence $\tau$ diverges linearly on SFNs with $\gamma>3$ and RNs, while it saturates for $\gamma \leq 3$. The scaling behavior of $\tau$ means that $S(t)$ on RNs and SFNs with $\gamma>3$ is nonzero constant in thermodynamic limit of $N \rightarrow \infty$, and exponentially decays to zero on SFNs with $\gamma \leq 3$. Hence we have

$$
\lim _{N \rightarrow \infty} S(N, t)= \begin{cases}S_{o} e^{-t / \tau_{\infty}} & (\gamma \leq 3) \\ S_{\infty} & (\gamma>3)\end{cases}
$$

where $\tau_{\infty}$ is the asymptotic value of $\tau$, and depends on $\gamma$. As $S(t)$ decays exponentially to zero for $\gamma \leq 3$, two particles initially located on the same node should meet or aggregate within finite time interval. Hence the finite life time on SFNs with $\gamma \leq 3$ supports the numerically expected condensed phase for any $\rho>0$ on SFNs with $2 \leq \gamma \leq 3$ as mentioned in the previous section. On the other hand, the finite $S_{\infty}$ on RNs and SFNs with $\gamma>3$ implies the existence of the fluid phase at $\rho \rightarrow 0$ limit, which is also consistent with the phase diagrams of Fig. 3 .

\section{SCA MODEL AT $\omega=\infty$}

At $\omega=\infty$, SCA model corresponds to ZRP with constant hopping rate on networks. On SFNs with $\gamma>2$, it was shown that ZRP undergoes complete condensation for any $\rho>0$ [6]. In condensation phenomena of ZRP on SFNs, the average mass at a node with degree $k, m_{k}$ in the steady state exhibits a discontinuous jump at a hub node. In ZRP with constant chipping rate for example, $m_{k}$ linearly increase until $k<k_{h u b}$ and jumps to the value $m^{*} \approx \rho N$ at $k=k_{h u b}[\underline{6}]$. The $k_{h u b}$ is the degree of the hub node which has the largest number of links.

To see whether the condensation occurs on RNs in ZRP, we measure $m_{k}$ for $\rho=0.4$ and 3.0 on RNs with $N=10^{4}$. As shown in the bottom inset of Fig. 6 (a), $m_{k}$ increases linearly in $k$ and finally jumps at $k_{h u b}$ as in ZRP on SFNs. For a fixed $N$, the mass of $k_{h u b}, m_{h u b}$ decreases as $\rho$ goes to zero as shown in the inset of Fig. 6 (a). So it is hard to observe the condensation at low density for small $N$. Since the complete condensation of ZRP on networks mainly comes from the inhomogeneity of degree distribution rather than the competition between dynamical processes, ZRP on RNs should exhibit the complete condensation for any $\rho>0$ as on SFNs. At $\omega=\infty$, we also measure $m_{k}$ on SFNs with $\gamma \leq 3$ and confirm the complete condensation as shown in Ref. [6]. Together with the recent study on SFNs [6], the complete condensation always occurs on both RNs and SFNs in zero range process with constant hopping rate. 
The discontinuity of $m_{k}$ clearly comes from the nature of chipping process of unit mass. That is, unit mass hops around according to the steady state distribution of Eq. (5), and then there is enough net particle current into the hub nodes to form infinite aggregation. However when the diffusion of the whole mass of each node turns on, the aggregation at the hub node diffuses around all nodes. The diffusion of the condensate completely change the behavior of $m_{k}$ in the condensed phase of finite $\omega . m_{k}$ linearly increase up to $k_{h u b}$ without any jump in the condensed phase of $\omega \neq \infty$. In next section, we numerically and analytically confirm the linearity of $m_{k}$ in $k$.

\section{AVERAGE MASS OF A NODE WITH DEGREE K}

Another interesting quantity in condensation phenomena on networks is the average mass at a node with degree $k, m_{k}$ in the steady state. In ZRP with chipping rate $u(m) \sim m^{\delta}$ on SFNs [6], the complete condensation takes place for $\delta<\delta_{c}=1 /(\gamma-1)$. For $\delta<\delta_{c}, m_{k}$ linearly increases with $k$ for $k<<k_{c}$, and algebraically increases as $k^{1 / \delta}$ for $k \geq k_{c}$. Especially at $\delta=0, m_{k}$ linearly increases until $k<k_{h u b}$ and jumps to the value $m^{*} \approx \rho N$. The $k_{h u b}$ is the degree of the hub node which has the largest number of links. The same type of behavior on $\mathrm{RN}$ for $\omega=\infty$ is shown in the inset of Fig. 6 (a).

In SCA model, as all masses can perform random walks according to the steady state distribution $P_{i}^{\infty}$ of Eq. (5), one can expect the jump of $m_{k}$ at $k_{h u b}$ in the condensed phase for $\omega \neq \infty$ in SCA model as in ZRP. However as shown in the main plot of Fig. 6 (a), $m_{k}$ increases linearly in $k$ up to $k_{h u b}$ and there is no jump unlike in ZRP. In Fig. 6 (a), we plot $m_{k}$ versus $k$ for $\rho=3.0$ and $\omega=1.0$ which corresponds to the condensed phase of both RN and SFN with $\gamma=4.3$. We also measure $m_{k}$ in the fluid phase $(\rho=0.2, \omega=1.0)$ of the both networks (not shown), and confirm $m_{k} \sim k$ without any jumps. For SFN with $\gamma=2.4, m_{k}$ also shows the linearity in $k$ for $\rho=3.0, \omega=1.0$. Our simulation results imply that the relation $m_{k} \sim k$ is valid for any $\rho>0$ in RNs and SFNs with $\gamma \geq 2$ for finite diffusion rate. The diffusion of masses indeed results in the linearity of $m_{k}$ in $k$ for any $\omega \neq \infty$.

In the steady state, as masses can perform random walks with a finite rate, the mass of a hub node $\left(m_{h u b}\right)$ diffuses to different nodes with the probability of being at node $i$ given as Eq. (5) [19]. The inset of Fig. 6 (b) shows the snapshot of the mass distribution of nodes with degree $k$ for a single sample at time $t=4 \times 10^{5}$ on SFN with $\gamma=2.4$ for $\rho=3.0$ and $\omega=1.0$. As shown, there is a peak which may be formed at $k_{h u b}$ as in ZRP. However the peak of mass $m^{*}$ is not always located at $k_{h u b}$ but diffuse around nodes according to $P_{i}^{\infty}$ (Fig. 6 (b)). Hence by taking average, the peak soaks into the average mass $m_{k}$ unlike in ZRP where all samples have the peak at $k_{h u b}$. To see this more explicitly, we derive the relation, $m_{k} \sim k$, based on the assumption that the diffusion (the random walks of masses) is the only relevant physical factor to decide $P(m)$ in the steady state.

First, we consider the average total mass $M_{k}$ of nodes with degree $k$ defined as

$$
M_{k}=\sum_{m=0}^{\infty} m P_{\infty}(m, k),
$$

where $P_{\infty}(m, k)$ is the probability of finding a random walker with mass $m$ at degree $k$ in $k$-space in the steady state. As mass distribution $P(m)$ in the steady state is independent of $k$, we have $P_{\infty}(m, k)=P(m) P_{k}^{\infty}$. $P_{k}^{\infty}$ is the probability of finding a random walker at a node with degree $k$ on the network. Then using $P_{i}^{\infty}$ of Eq. (5), we write $P_{k}^{\infty}$ as

$$
P_{k}^{\infty}=\sum_{i=1}^{N} P_{i}^{\infty} \delta_{k_{i}, k}=\frac{k N P(k)}{\sum_{i=1}^{N} k_{i}},
$$

where $P(k)$ is a degree distribution. From Eq. (77) and (8), we have

$$
M_{k}=\left[\frac{k N P(k)}{\sum_{i=1}^{N} k_{i}}\right]\left[\sum_{m=0}^{\infty} m P(m)\right] .
$$

So $M_{k}=\bar{m} k P(k) /<k>\sim k P(k)$. Then the average mass of a node with degree $k$ is given as

$$
m_{k}=\frac{M_{k}}{N P(k)} \sim k / N .
$$

As expected, $m_{k}$ scales as $m_{k} \sim k$ for any degree distribution $P(k)$. As Eq. (10) is valid for any density $\rho>0, m_{k}$ does not undergoes the condensation transitions unlike $P(m)$ of Eq. (1). Therefore the steady state distribution of $m_{k}$ is determined by diffusion of masses rather than chipping of unit mass unlike in ZRP.

\section{SUMMARY}

We investigate the condensation phase transitions of symmetric conserved-mass aggregation (SCA) model on networks. In SCA model, masses diffuse with unite rate, and unit mass chips off from mass with rate $\omega$. SCA model undergoes condensation phase transitions via the competition between diffusion and chipping processes [13].

First we consider the $\omega \neq \infty$ case. On random and scale-free networks of $\gamma>3$, SCA model undergoes the same type of condensation transitions from fluid phase into condensed phase as in one dimensional lattice of Ref. [13]. However unlike on regular lattices, the critical line on the networks depends on the network structures. On the other hand, on scale-free networks of $\gamma \leq 3$ where one 
or several nodes have a finite fraction of degrees, an infinite aggregation with exponentially decaying background mass distribution always takes place for any nonzero density, so no phase transitions occur for $\omega \neq \infty$. The condensation and exponential mass distribution of small masses come from the generic inhomogeneity of network structure of SFNs with $\gamma \leq 3$. However we are not able to numerically show the existence of the condensation phase at zero density limit for $\gamma \leq 3$ due to the small world nature of networks [5]. Instead we numerically study the survival probability of a particle in pair annihilating random walks, so-called one lamb and one lion problem on networks.

For the formation of an infinite aggregation of masses at zero density limit, unit mass chipped off from the infinite aggregation should aggregate again with the aggregation within finite time interval. We numerically show that in thermodynamic limit, the survival probability $S(t)$ of a lamb (unit mass) is finite on random networks and scale-free networks (SFNs) with $\gamma>3$, but exponentially decays to zero with finite life time on SFNs with $\gamma \leq 3$. Based on the finite life time of a lamb on SFNs with $\gamma \leq 3$, we indirectly confirm the incomplete condensed phase for any $\rho>0$ on SFNs with $\gamma \leq 3$.

At $\omega=\infty$, SCA model corresponds to zero-range process (ZRP) with constant chipping rate [1]. ZRP with constant chipping rate on SFNs was shown to exhibit complete condensation [6]. We also numerically show that the complete condensation takes place for any $\rho>0$ on random networks by measuring average mass of a node with degree $k$. Hence the complete condensation always takes place for any nonzero density in ZRP with constant chipping rate on random and scale-free networks.

Finally we investigate the behavior of the average mass of a node with degree $k, m_{k}$ in the fluid and the condensed phase. In ZRP with constant chipping rate, $m_{k}$ linearly increases with degree $k$, and jumps to the total mass of the system at hub degree $k_{h u b}$ [ $]$. Hence in SCA model, $m_{k}$ is expected to show such a jump in the condensed phase for $\omega \neq \infty$. However $m_{k}$ linearly increases up to $k_{h u b}$ without jumps in both the fluid and the condensed phase. We numerically confirm the linearity of $m_{k}$, and also analytically show $m_{k} \sim k$ with the assumption that the diffusion is the only relevant factor in the steady state. Therefore the steady state distribution of $m_{k}$ is determined by diffusion of masses rather than chipping of unit mass unlike in ZRP.

This work is supported by Grant No. R01-2004-00010148-0 from the Basic Research Program of KOSEF. We also thank Prof. Soon-Hyung Yook for critical reading of our manuscript.
[1] M. R. Evans, Braz.J.Phys. 30, 42 (2000); M. R. Evans and T. Hanney, J. Phys. A 38, R195 (2005).

[2] M. R. Evans, Europhys. Lett. 36, 13 (1996).

[3] O. J. O'Loan, M. R. Evans, and M. E. Cates, Phys. Rev. E 58, 1404 (1998).

[4] D. van der Meer et al., J. Stat. Mech. Theor. Exp. 04, P04004 (2004); J. Torok, cond-mat/0407567

[5] S. N. Dorogovtsev and J. F. F. Mendes, Adv. Phys. 51, 1079 (2002); R. Albert and A.-L. Barabási, Rev. Mod. Phys. 74, 47 (2002)

[6] J. D. Noh, G. M. Shim, and H. Lee, Phys. Rev. Lett. 94, 198701 (2005).

[7] A. G. Angel, T. Hanney, and M. R. Evans, condmat/0509238.

[8] R. M. Ziff, J. Stat. Phys. 23, 241 (1980).

[9] W. H. White, J. Colloid Interface Sci. 87, 204 (1982).

[10] A. E. Scheidegger, Bull. I.A.S.H. 12, 15 (1967).

[11] A. Maritan, A. Rinaldo, R. Rigon, A. Giacometti, and I. R. Iturbe, Phys. Rev. E 53, 1510 (1996); M. Cieplak, A. Giacometti, A. Maritan, A. Rinaldo, I. R. Iturbe, and J. R. Banavar, J. Stat. Phys. 91, 1 (1998).

[12] S. K. Friedlander, Smoke, Dust and Haze (Wiley Interscience, New York, 1977).

[13] S. N. Majumdar, S. Krishnamurthy, and M. Barma, Phys. Rev. Lett. 81, 3691 (1998); S. N. Majumdar, S. Krishnamurthy, and M.Barma, J. Stat. Phys. 99, 1 (2000).

[14] R. Rajesh and S. N. Majumdar, Phys. Rev. E 63, 036114 (2001).

[15] R. Rajesh and S. Krishnamurthy, Rhys. Rev. E 66, 046132( 2002).

[16] R. Rajesh, D. Das, B. Chakraborty, and M. Barma, Phys.
Rev. E 66, 056104(2002).

[17] A. V. Goltsev, S. N. Dorogovtsev, and J. F. F. Mendes, Phys. Rev. E 67, 026123 (2003).

[18] K. -I. Goh, B. Kahng and D. Kim, Phys. Rev. Lett. 87, 278701 (2001).

[19] J. D. Noh and H. Rieger, Phys. Rev. Lett. 92, 118701 (2004).

[20] H. Takayasu, I. Nishikawa, and H. Tasaki, Phy. Rev. A 37, 3110 (1988); H. Takayasu, Rhys. Rev. Lett, 63, 2563 (1989).

[21] R. Rajesh and S. N. Majumdar, Phys. Rev. E 63, 036114 (2001).

[22] X. Campi and H. Krivine, Nucl. Phys. A 620, 46 (1997).

[23] M. Bramson and D. Griffeath, "Capture problems for coupled random walks", in Random walks, Brownian motion, and interacting particle systems: a festschrift in honor of Frank Spitzer, eds. R.Durrett and H.Kesten, (Boston, Birkhauser, 1991), 153-188.

[24] W. Feller, An Introdution to Probability Theory (Wiley, New Yourk, 1971), Vol. 1.

[25] Aspects and Applications of the Random Walk (NorthHolland, Amsterdam, 1994).

[26] P. L. Krapivsky and S.Redner, J. Phys. A 29, 5347 (1996). 

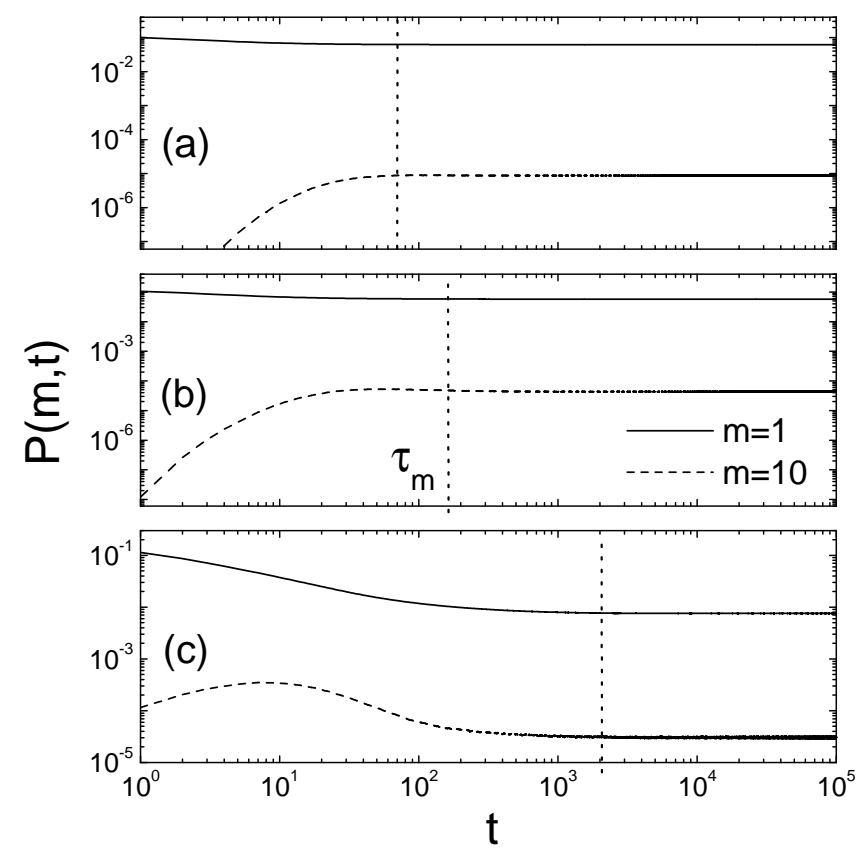

FIG. 1: $P(m, t)$ of $m=1$ and 10 for $\rho=0.1$ and $\omega=1$ on networks of $N=10^{4}$. Each panel corresponds to RN (a), SFN with $\gamma=4.3$ (b) and $\gamma=2.4$ (c). The vertical dotted line denotes the characteristic time $\tau_{m}$. 


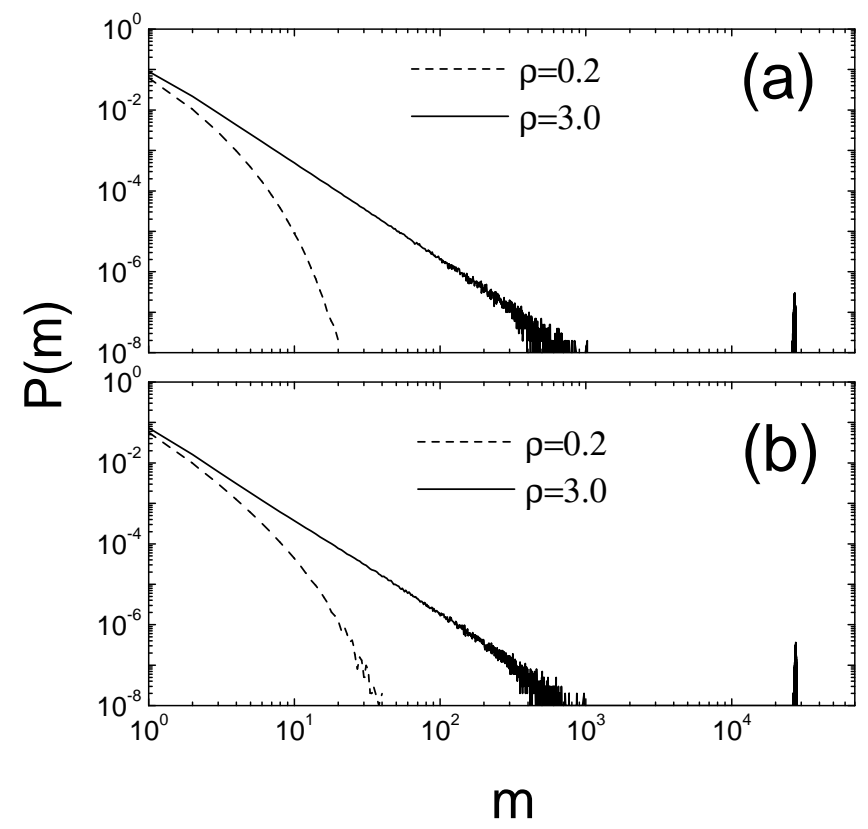

FIG. 2: $P(m)$ on RN (a) and SFN with $\gamma=4.3(\mathrm{~b})$ at $\omega=1.0$. In each panel, the solid and the dashed line correspond to $P(m)$ of $\rho=3.0$ (condensed phase) and 0.2 (fluid phase) respectively.

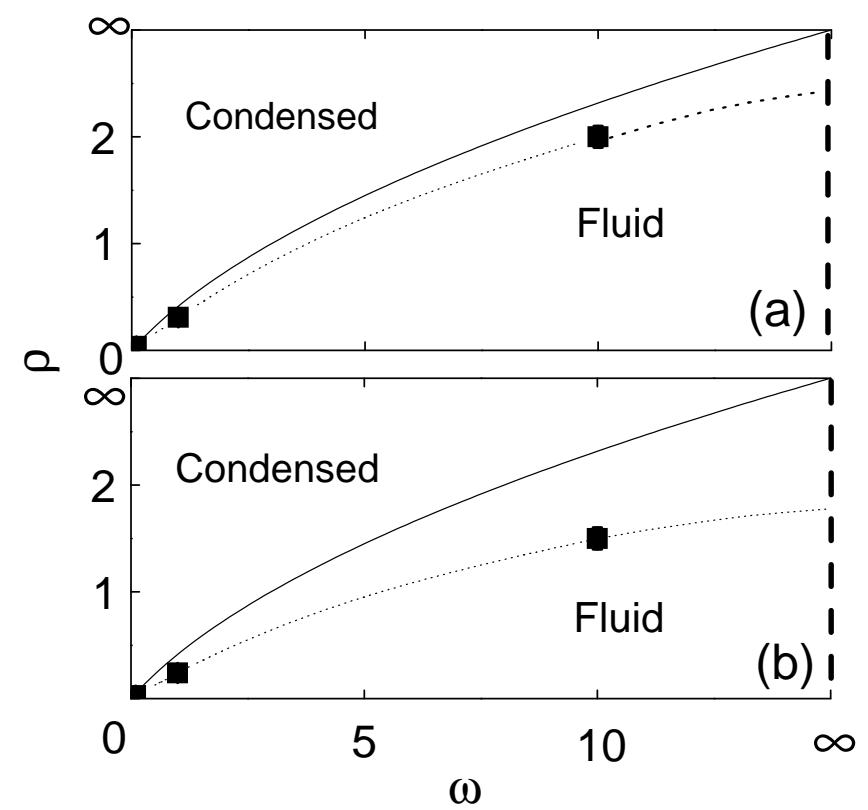

FIG. 3: The $\rho-\omega$ phase diagram of RN (a) and SFN with $\gamma=4.3$ (b). In each panel, the solid line is the critical line of $\rho_{c}(\omega)=\sqrt{\omega+1}-1$ on regular lattices of Ref. [13]. The dotted line between data points is a guide to the eye. The dashed line denotes $\omega=\infty$ line. 


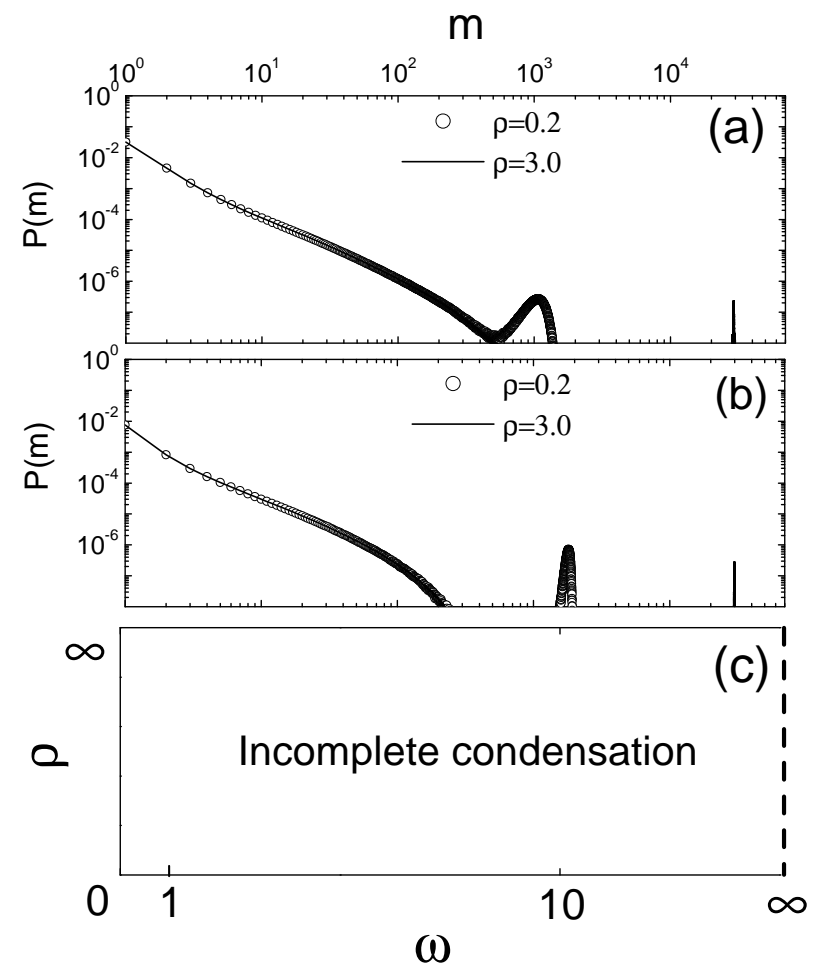

FIG. 4: $\quad P(m)$ on SFN with $\gamma=3$ (a) and $\gamma=2.4$ (b) at $\omega=1.0$. In each panel, the solid and the dashed line correspond to $P(m)$ of $\rho=3.0$ and 0.2 respectively. (c) the typical phase diagram of SFNs with $\gamma \leq 3$. The dashed line denotes the line of $\omega=\infty$.

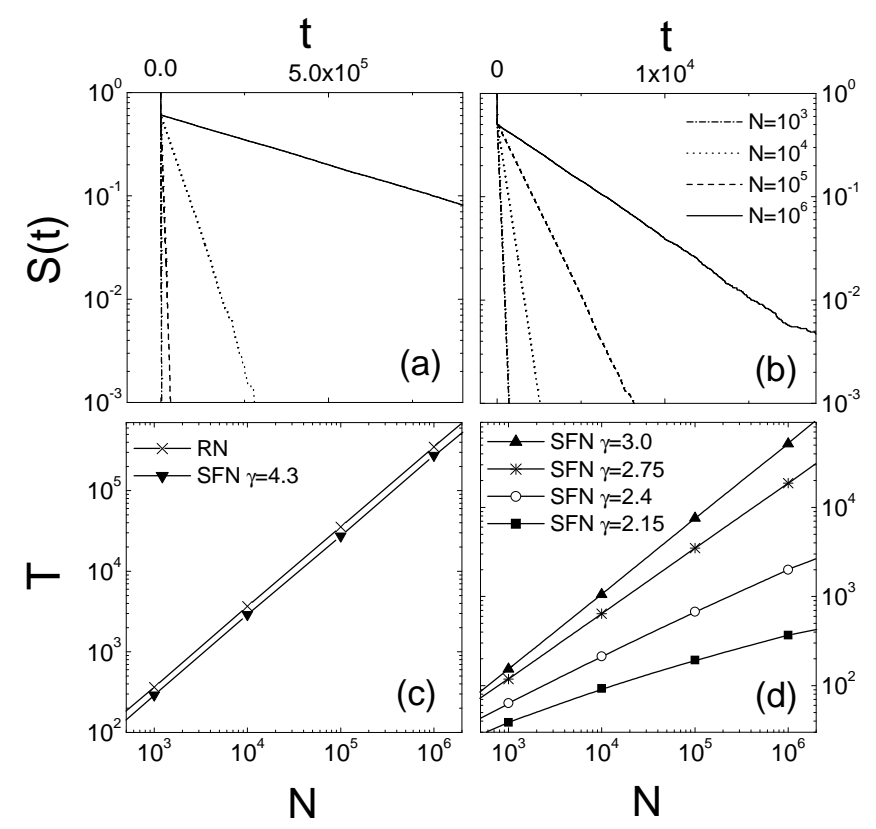

FIG. 5: Semi-log plot of the survival probability $S(t)$ on SFN with $\gamma=4.3$ (a) and 2.4 (b) of various size $N$ up to $10^{6}$. (c) The average life time $T$ on RN and SFN with $\gamma=4.3$. (d) $T$ on SFNs with $\gamma \leq 3$. The solid line between data points is a guide to the eye. 

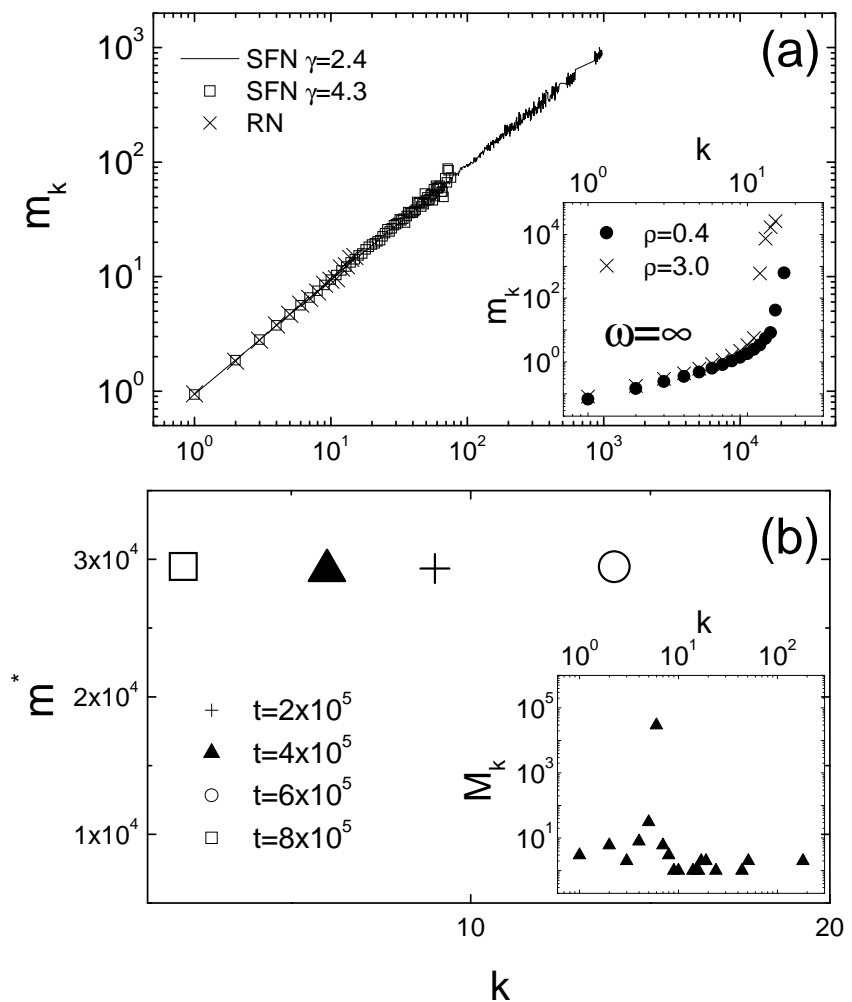

FIG. 6: (a) The main plot shows $m_{k}$ of Eq. (10) for $\rho=3.0$ and $\omega=1$ on RN, SFN with $\gamma=4.3$ and 2.4 in condensed phase. The inset shows $m_{k}$ at $\omega=\infty$ on RN. (b) $M_{k}$ of a single sample on SFN with $\gamma=2.4$ at different time for $\rho=3$ and $\omega=1$ of the condensed phase. The main plot only shows the maximum mass $m^{*}$ of $M_{k}$ of a single sample at different time steps, and the inset shows the snapshot of the $M_{k}$ at $t=4 \times 10^{5}$. 